\title{
A Novel Species-Level Group of Streptomyces Exhibits Variation in Phytopathogenicity Despite Conservation of Virulence Loci
}

\author{
Alexandra J. Weisberg, ${ }^{1}$ Charles G. Kramer, ${ }^{2}$ Raghavendhar R. Kotha, ${ }^{3}$ Devanand L. Luthria, ${ }^{3}$ \\ Jeff H. Chang, ${ }^{1,4}$ and Christopher R. Clarke ${ }^{2,+}$ \\ ${ }^{1}$ Department of Botany and Plant Pathology, Oregon State University, OR 97331, U.S.A. \\ ${ }^{2}$ Genetic Improvement for Fruits and Vegetables Lab, Beltsville Agricultural Research Center, Agricultural Research Service, \\ USDA, Beltsville, MD 20705, U.S.A. \\ ${ }^{3}$ Food Composition and Methods Development Lab, Beltsville Human Nutrition Research Center, Agricultural Research \\ Service, USDA, Beltsville, MD 20705, U.S.A. \\ ${ }^{4}$ Center for Genome Research and Biocomputing, Oregon State University, OR 97331, U.S.A.
}

Accepted 6 October 2020.

\begin{abstract}
The genus Streptomyces includes several phytopathogenic species that cause common scab, a devastating disease of tuber and root crops, in particular potato. The diversity of species that cause common scab is unknown. Likewise, the genomic context necessary for bacteria to incite common scab symptom development is not fully characterized. Here, we phenotyped and sequenced the genomes of five strains from a poorly studied Streptomyces lineage. These strains form a new specieslevel group. When genome sequences within just these five strains are compared, there are no polymorphisms of loci implicated in virulence. Each genome contains the pathogenicity island that encodes for the production of thaxtomin A, a phytotoxin necessary for common scab. Yet, not all sequenced strains produced thaxtomin A. Strains varied from nonpathogenic to highly virulent on two hosts. Unexpectedly, one strain that produced thaxtomin $A$ and was pathogenic on radish was not aggressively pathogenic on potato. Therefore, while thaxtomin A biosynthetic genes and production of thaxtomin A are necessary, they are not sufficient for causing common scab of potato. Additionally, results show that even within a species-level group of Streptomyces strains, there can
\end{abstract}

The 16S rDNA sequence data (independently Sanger sequenced) are available under GenBank accession numbers MT360635 to MT360639. The genome sequence short reads and assemblies are available at the National Center for Biotechnology Information under Bioproject PRJNA640830.

${ }^{\dagger}$ Corresponding author: C. R. Clarke; christopher.clarke@usda.gov

Funding: Funding for the Clarke lab was provided by the United States Department of Agriculture (USDA) Agricultural Research Service CRIS 8042-21000-283. A. J. Weisberg was supported by USDA National Institute of Food and Agriculture award 2017-67012-26126. The funders had no role in study design, data collection and analysis, decision to publish, or preparation of the manuscript.

*The $\boldsymbol{e}$-Xtra logo stands for "electronic extra" and indicates there are supplementary figures and table files published online.

The author(s) declare no conflict of interest.

This work was produced by an employee of the federal government as part of their official duties and is therefore in the public domain, 2021. be aggressively pathogenic and nonpathogenic strains despite conservation of virulence genes.

Keywords: common scab, evolution, pathogenicity islands, Streptomyces, thaxtomin, virulence

Common scab is a major disease of potato present in all growing regions in the world. The disease manifests as raised or pitted scab lesions on the surface of tubers (Braun et al. 2017). Common scab is known to be caused by a Streptomyces species (Francis et al. 2010; Thapa et al. 2019). The Streptomyces genus is an incredibly diverse genus of actinobacteria with more than 800 named species with standing in nomenclature (LPSN website), but only 14 Streptomyces species are known to have members that are plant pathogens. This diversity of both pathogenic and nonpathogenic species of Streptomyces is a challenge for the meaningful characterization of local Streptomyces populations.

Resolving the genetic diversity of Streptomyces spp. is important for understanding the evolution of common scab pathogens. 16S rDNA sequences are frequently used but are often not sufficient to delineate Streptomyces species (Labeda et al. 2012). Use of multilocus sequence analysis (MLSA) has been more successful for classifying Streptomyces strains (Labeda 2011; Labeda et al. 2017). However, phylogenetic analyses using both MLSA and whole-genome sequence data indicate that many Streptomyces strains are misclassified and may result in misleading conclusions on the evolution of phytopathogenicity in the genus (Bouchek-Mechiche et al. 2006; Thapa et al. 2019; Zhang et al. 2016). These inconsistencies have yet to be resolved. Robust analysis of whole-genome sequence data may be used to characterize the diversity of phytopathogenic Streptomyces strains. But, of the several thousand publicly available Streptomyces genome sequences, only a handful correspond to phytopathogenic Streptomyces strains. Furthermore, due to an abundance of repetitive regions, transposons, and other mobile genetic elements that are common in Streptomyces genomes, most publicly available assemblies are not of sufficient quality (Studholme 2016).

Horizontal gene transfer is hypothesized to be central to common scab and the ability to identify instances of horizontal transfer requires a robust evolutionary framework. All 


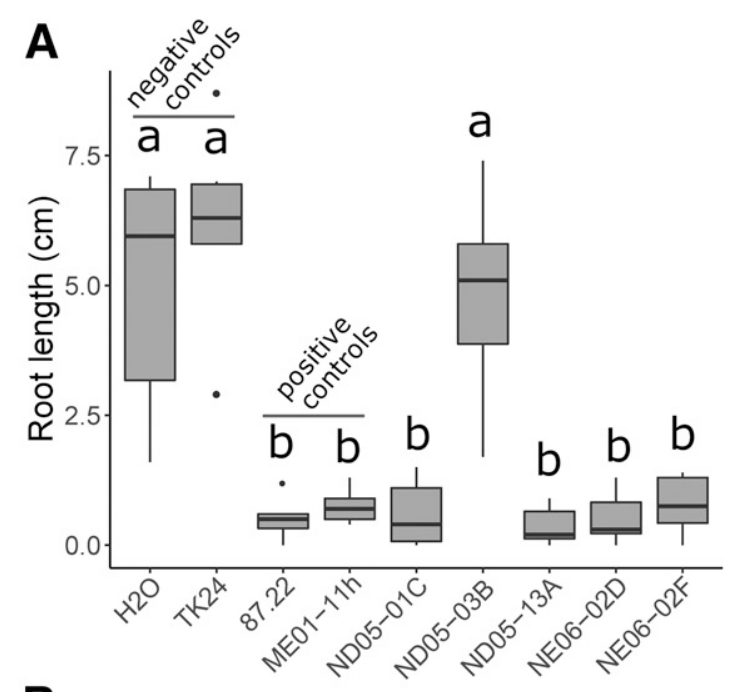

B
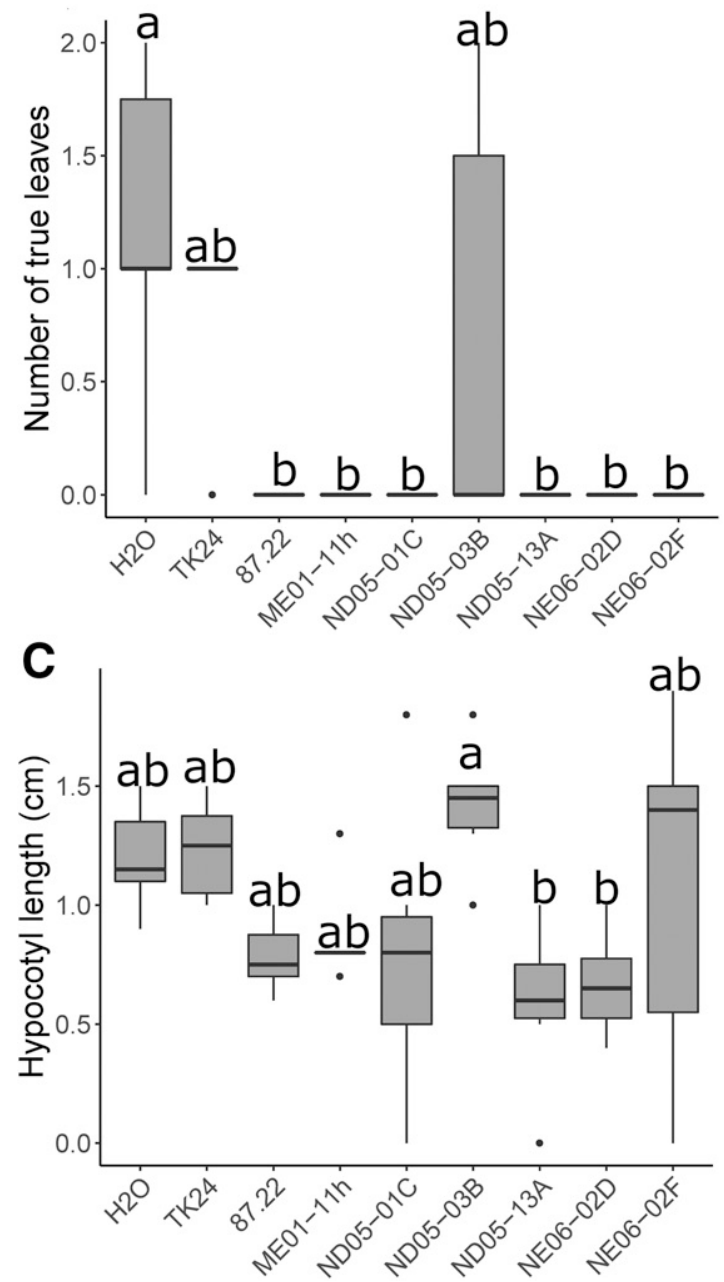

Fig. 1. Four of the five tested strains in the new species group are pathogenic to radish. Radish seedlings were mock-inoculated with water or Streptomyces strains grown in thaxtomin-inducing oat bran broth media. Treated plants were quantified for $\mathbf{A}$, root length, $\mathbf{B}$, number of true leaves, and $\mathbf{C}$, hypocotyl length. Boxplots indicate the median, interquartile range, minimum, maximum, and outliers (small black points) of six replicate plants. Box plots sharing the same letter are not significantly different based on Tukey's honest significant difference $(P<0.05)$ grouped within each media type. Similar results were obtained in three independent experiments.
Streptomyces strains known to cause common scab produce the phytotoxin thaxtomin A, which inhibits cellulose synthesis of the host and is considered necessary for virulence (Bignell et al. 2010a; Fry and Loria 2002; Healy et al. 2000; Loria et al. 2008). Thaxtomin A production is encoded within a pathogenicity island (PAI) (Bukhalid and Loria 1997; Kers et al. 2005).This PAI is hypothesized to be horizontally transferred among Streptomyces strains and necessary for the emergence of new lineages of common scab pathogens (Bukhalid et al. 2002; Zhang et al. 2016, 2018). Other genes, such as NECl (Joshi et al. 2007a), TOMA (Seipke and Loria 2008), and the coronafacic acid biosynthetic gene cluster (Bignell et al. 2010b), have been suggested to contribute to pathogenicity to a lesser extent.

The PAI is hypothesized to circumscribe most genes necessary for regulating and producing thaxtomin. Expression of the thaxtomin biosynthetic genes is tightly regulated, which is speculated to ensure that the transition from saprophyte to pathogen occurs only in the presence of a potential host plant (Jourdan et al. 2017). The thaxtomin biosynthetic operon TXTAB is expressed only in the presence of cellobiose and cellotriose, hypothesized to be derived from the degradation of plant cells (Johnson et al. 2007; Lerat et al. 2010; Wach et al. 2007) due to de-repression of the cellulose utilization protein CebR from the two CebR binding sites in the PAI (Francis et al. 2015). This results in the upregulation of TXTAB and the transcriptional regulatory gene TXTR (Joshi et al. 2007b). Thaxtomin production is further regulated through the action of several global regulatory BLD genes (Bignell et al. 2014).

To build a resource for understanding the genetic diversity of phytopathogenic Streptomyces strains, several hundred Streptomyces strains have been isolated from potato tubers exhibiting common scab symptoms (Wanner 2006, 2009). One new Streptomyces 'ribotype' (informally called 3,6,7) containing the TXTAB operon was identified and described as Streptomyces bottropensis-like (Wanner 2009). The S. bottropensis type strain ATCC25435 is a nonpathogenic strain; therefore, the discovery of this ribotype suggested that the $S$. bottropensis species has the potential to horizontally acquire the PAI and transition to being a pathogen of plants. More recently, pathogenic strains classified as $S$. bottropensis isolates have been identified in Spain and China (Sarwar et al. 2018; Zhou et al. 2017).

Here, we combined assays for thaxtomin and virulence with analyses of whole-genome sequences to characterize the genetic and phenotypic diversity of the poorly understood 3,6,7 lineage. Findings show that each of the five strains has the same set of homologs of genes implicated in Streptomyces virulence. Unexpectedly, the strains varied in their ability to incite disease and also differed in host range. Analysis of whole genome sequences showed the five strains are more distantly related to S. bottropensis and represent a new species group. Collectively, findings contribute toward a genomic and evolutionary framework for studying plant-Streptomyces interactions.

\section{RESULTS}

\section{Only a subset of the strains from ribotype $3,6,7$ are plant pathogens.}

Each of the five ribotype 3,6,7 strains are predicted to be pathogenic on plants. Previously reported findings from PCR analysis using TXTAB-specific primers (Wanner 2009) indicated that all five ribotype 3,6,7 strains harbor the thaxtomin biosynthetic genes. Therefore, we expected each of the strains in this clade to be plant pathogens. To test this hypothesis, we inoculated radish seedlings with mycelial suspensions of the strains grown in either thaxtomin-inducing oat bran broth 
(OBB) or non-thaxtomin inducing yeast-malt extract (YME) and quantified treatment-induced stunting of the radish seedlings.

Unexpectedly, one of the tested strains, ND05-03B, was not pathogenic to radish seedlings. The other four ribotype 3,6,7 strains (ND05-01C, ND05-13A, NE06-02D, NE06-02F) were pathogenic to radish following inoculation with mycelia grown in OBB media, as quantified by stunting of root growth (Fig. 1A). Those four strains caused levels of stunting of root growth similar to those of the known aggressive S. scabiei strains 87.22
(Bignell et al. 2010b) and ME01-11h (Wanner 2004; Clarke et al. 2019), which served as the positive controls. Strain ND0503B did not attenuate root growth, and plants were similar to those in the water control and the treatment group with the nonpathogenic $S$. lividans TK24 that does not harbor the thaxtomin biosynthetic gene cluster (Rückert et al. 2015), which served as the negative controls. Stunting of radish growth as quantified by development of a number of true leaves also demonstrated that ND05-03B was not pathogenic on radish, while the other tested strains are pathogenic (Fig. 1B). The
A

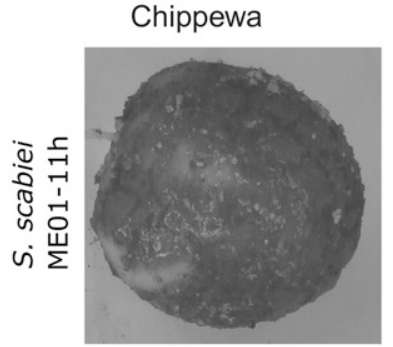

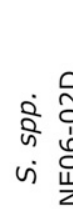

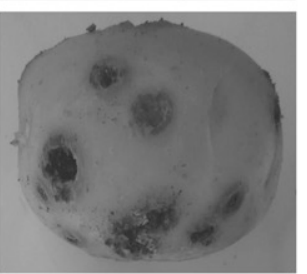

ì

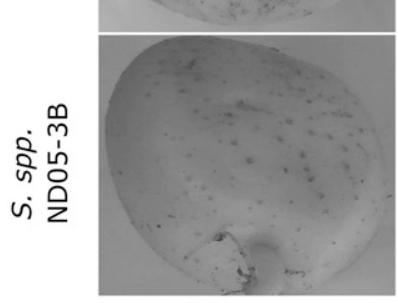

B

Chippewa
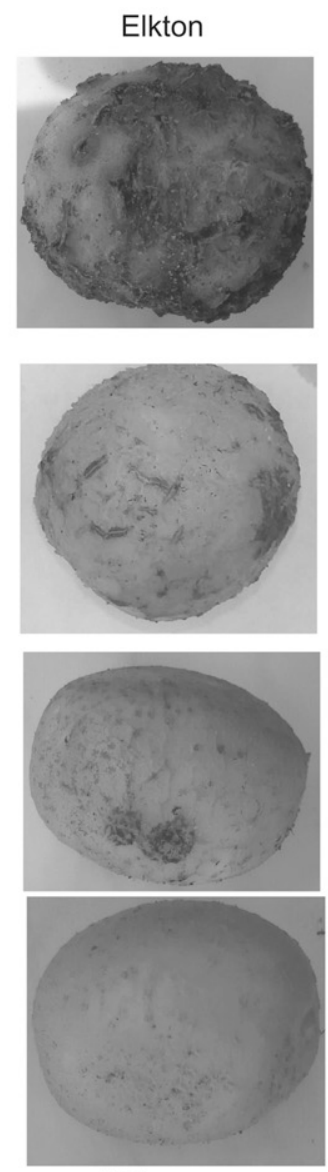

Elkton
Green Mountain
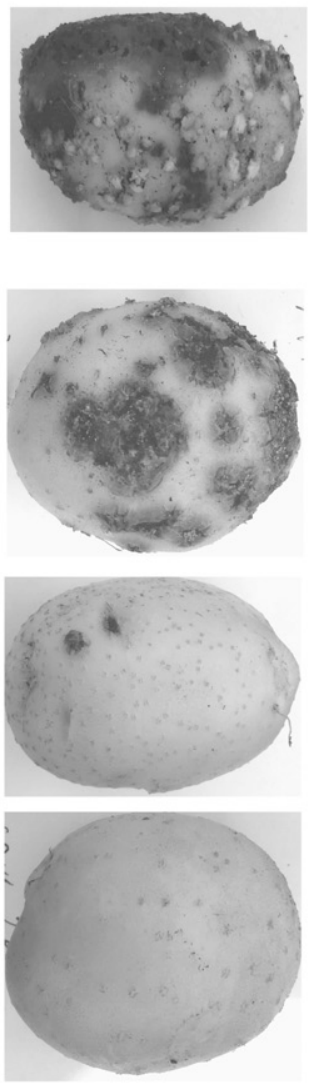

Green Mountain
Payette Russet
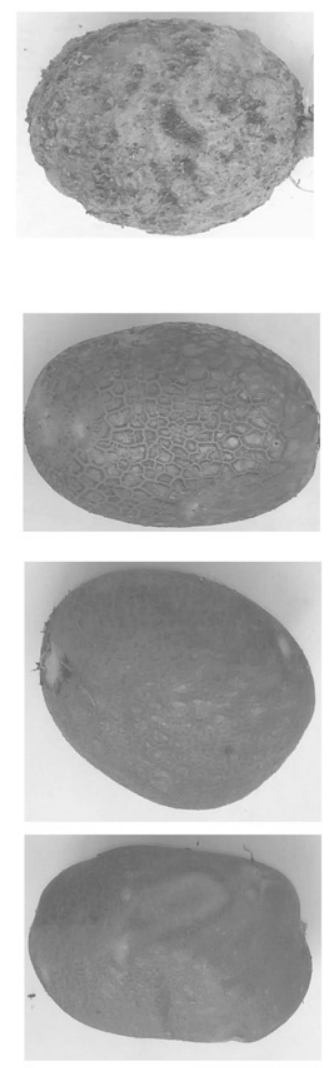

Payette Russet

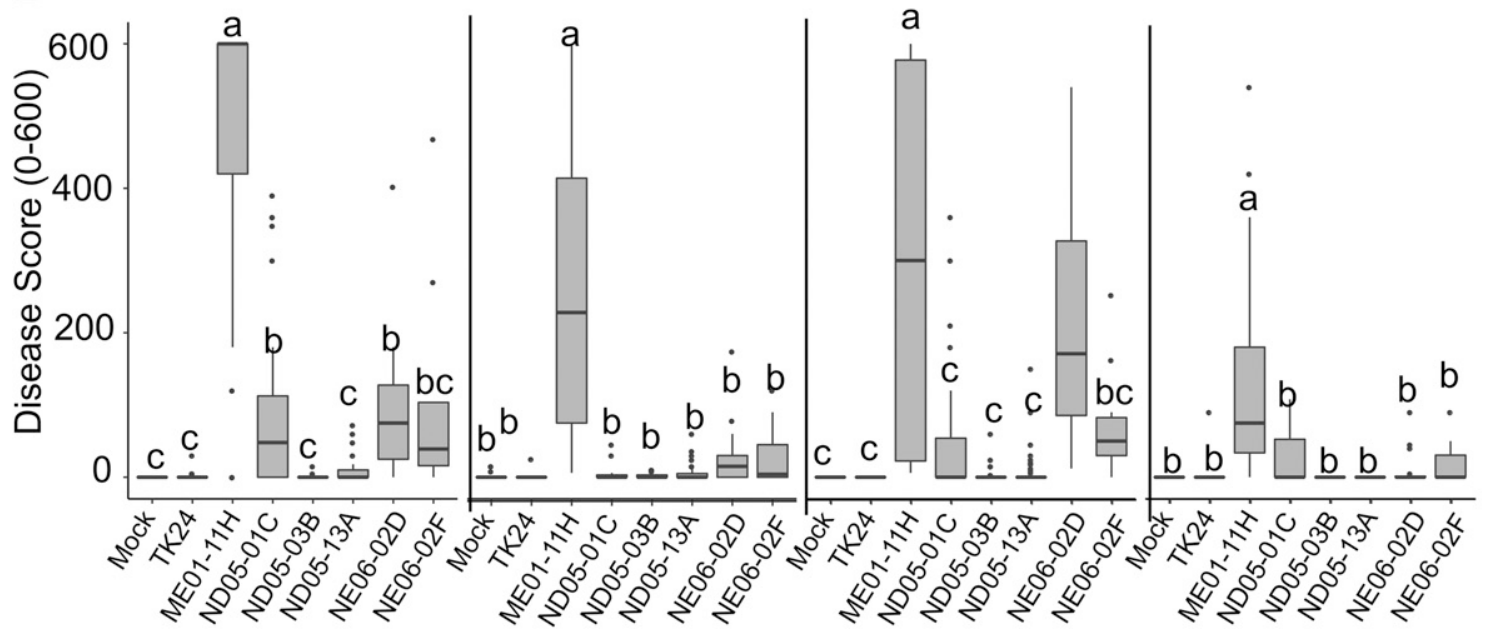

Fig. 2. Three of the five tested strains in the new species group are pathogenic to potato. A, Pictures of potatoes representative of those with the most severe disease symptoms. B, Summary of disease severity ratings of four potato cultivars infected with Streptomyces strains. Boxplots indicate the median, interquartile range, minimum, maximum, and outliers (small black points) of eight replicate pots with an average of four tubers sampled from each pot (all tubers $>2 \mathrm{~g}$ were sampled). Box plots sharing the same letter are not significantly different based on Tukey's honest significant difference $(P<0.05)$ grouped by each potato cultivar. 
evidence for differential pathogenicity among the tested strains was less clear when using radish hypocotyl length as the phenotype for radish stunting, but the same general trend was observed (Fig. 1C). There were significant differences among group means based on two-way analysis of variance (ANOVA) of root length, hypocotyl length, and number of true leaves, with Streptomyces strain being a significant factor for all three measured dependent variables (Supplementary Table S1). When Streptomyces mycelial suspensions were produced in YME medium prior to inoculation, growth attenuation was less severe for all pathogenic strains, but the same trends were nevertheless observed. Strain ND05-03B, unlike others, did not attenuate radish growth (Supplementary Fig. S1). Streptomyces growth media (YME versus OBB) significantly affected the number of true leaves and root length, based on ANOVA, but there was no Streptomyces strain by media type interaction (Supplementary Table S1).

Similarly, we were also surprised to have observed variation in virulence on potato. Only three of the five strains (ND0501C, NE06-02D, NE06-02F) are aggressive pathogens on any of the four tested potato cultivars (Fig. 2; Supplementary Fig. S2). We used soil inoculation of Solanum tuberosum plants growing in pots in a greenhouse to assess virulence of strains on potato. Cultivars Green Mtn and Chippewa are considered common scab-susceptible, cultivar Elkton is considered moderately resistant, and cultivar Payette Russet is considered resistant (Clarke et al. 2019). There were significant differences among group means based on two-way ANOVA of the disease severity ratings of the tubers, with both cultivar and Streptomyces treatment being significant factors and a significant strain by cultivar interaction (Supplementary Table S2). The strains ND05-03B and ND05-13A did not cause significant common scab symptoms on any of the tested potato cultivars, and tubers were similar to those in mock controls and those treated with the nonpathogenic TK24 isolate.

\section{Ribotype 3,6,7 is a novel species-level group.}

The dramatic variation in virulence could reflect the possibility that strains represent different species groups and differ extensively in their genomes and/or the thaxtomin PAI. Therefore, we sequenced and compared the genomes of the five ribotype 3,6,7 strains (Table 1).

Surprisingly, the five strains formed a single, novel specieslevel group. We concatenated the gene sequences of atpD, recA, gyrB, rpoB, and $\operatorname{trpB}$, commonly used for Streptomyces MLSA (Labeda 2011; Labeda et al. 2017), and constructed a phylogeny that included type strains of pathogenic and select nonpathogenic Streptomyces strains (Fig. 3A). The five sequenced strains do not cluster with the $S$. bottropensis type strain, indicating that the earlier designation of these strains as
S. bottropensis-like was misleading. The five sequenced strains formed a monophyletic clade distinct from any of the pathotype Streptomyces strains. Streptomyces strains faxanaA7 and CF124, with publicly available genome sequences, are also part of this novel species group (Supplementary Fig. S3). Findings from whole genome analysis were consistent with results from the MLSA phylogeny. These seven strains exceeded a threshold of $95 \%$ average nucleotide identity (ANI), used to operationally define species-level groups (Fig. 3B; Supplementary Fig. S5, Supplementary Table S3). It was also noted that in the phylogeny, several strains named as $S$. scabiei did not cluster with the S. scabiei type strain ATCC49173, and their classification should be revisited.

To understand the source for misclassifying the ribotype 3,6,7 strains as $S$. bottropensis-like, we constructed a phylogeny using the $16 \mathrm{~S}$ rDNA sequence. In the phylogeny, all five of these strains formed a monophyletic clade with the $S$. bottropensis type strain ATCC25435 (Supplementary Fig. S4), confirming that use of the $16 \mathrm{~S}$ rDNA sequence for inferring species delineations misled its classification.

\section{Only four of the five strains produce thaxtomin, despite all encoding the thaxtomin biosynthetic cluster.}

We next tested the hypothesis that phenotypic variation on plants is associated with variation in thaxtomin production. We quantified the amount of thaxtomin from each of the strains grown in thaxtomin-inducing OBB medium (Beauséjour et al. 1999) or thaxtomin-noninducing YME medium. Strains ND05-01C, ND05-13A, NE06-02D, and NE06-02F each produced thaxtomin when grown in OBB medium (Fig. 4A), though amounts detected were less than that of the highly aggressive S. scabiei isolate ME01-11h. The nonpathogenic isolate ND05-03B did not produce detectable levels of thaxtomin regardless of the media in which it was grown (Fig. 4A).

To identify the potential genetic basis for differences in thaxtomin production among the tested strains, we compared the sequences of the thaxtomin PAIs. We hypothesized that the thaxtomin PAI of strain ND05-03B would be divergent from the PAI present in the other four strains; however, the thaxtomin PAI of strain ND05-03B has no genetic differences relative to PAIs of strains that produce thaxtomin (Fig. 4B). Among the five strains, there is a large polymorphism in the thaxtomin PAI, but it does not correlate with the observed phenotypic variation. The $t x t$ regions of the nonpathogenic strain ND05-03B is similar to corresponding regions of ND05-01C, NE06-02D, and NE06-02F in having a $23-\mathrm{kb}$ transposon relative to the reference strain S. scabiei 87.22 (Fig. 4B). This insertion contains mostly repetitive elements and integrase/transposase annotated genes (Supplementary Table S4). This 23-kb insertion is absent from strain ND05-13A and the reference strain 87.22. In the

Table 1. Streptomyces strains used in this work and genome assembly statistics

\begin{tabular}{|c|c|c|c|c|c|c|c|c|}
\hline \multirow{2}{*}{$\begin{array}{l}\text { Strain } \\
\text { name }\end{array}$} & \multirow[b]{2}{*}{ Species } & \multicolumn{2}{|c|}{ Isolated in } & \multirow{2}{*}{$\begin{array}{l}\text { No. of } \\
\text { contigs }\end{array}$} & \multirow{2}{*}{$\begin{array}{l}\text { N50 contigs } \\
(\mathrm{kb})\end{array}$} & \multirow{2}{*}{$\begin{array}{c}\text { Total assembly } \\
\text { (kb) }\end{array}$} & \multirow[b]{2}{*}{ Reference } & \multirow{2}{*}{$\begin{array}{c}\text { GenBank genome } \\
\text { accession }\end{array}$} \\
\hline & & Year & State & & & & & \\
\hline ND05-01C & New & 2005 & North Dakota & 91 & 336 & 10,826 & This work & SAMN15335179 \\
\hline ND05-03B & New & 2005 & North Dakota & 65 & 458 & 10,572 & This work & SAMN15335181 \\
\hline ND05-13A & New & 2005 & North Dakota & 78 & 357 & 10,557 & This work & SAMN15335180 \\
\hline NE06-02D & New & 2006 & Nebraska & 74 & 480 & 10,938 & This work & SAMN15335182 \\
\hline NE06-02F & New & 2006 & Nebraska & 67 & 537 & 10,840 & This work & SAMN15335183 \\
\hline ME01-11h & S. scabiei & 2001 & Maine & N/A & N/A & N/A & $\begin{array}{c}\text { Wanner 2004; Clarke } \\
\text { et al. } 2019\end{array}$ & N/A \\
\hline 87.22 & S. scabiei & 1987 & Wisconsin & 1 & N/A & 10,149 & Bignell et al. 2010b & FN554889.1 \\
\hline TK24 & S. lividans & Unk. & Unk. & N/A & N/A & N/A & Rückert et al. 2015 & CP009124 \\
\hline
\end{tabular}


same genomic location, strains ND05-13A and 87.22, instead, have a $13-\mathrm{kb}$ region that contains different integrase genes as well as a gene predicted to encode a CHAT domain-containing protein.

We next used the sequence of the thaxtomin PAI of the reference genome sequence to identify single nucleotide polymorphisms (SNPs). Importantly, the thaxtomin PAI of the non-thaxtomin-producing strain ND05-03B is identical at single nucleotide level to that of the thaxtomin-producing strain ND05-01C. In addition, only one SNP distinguished the thaxtomin PAI of ND05-13A from that of the reference genome 87.22 (Supplementary Table S5). An additional 63 SNPs distinguished the PAIs of the other four strains from the 87.22 PAI. However, 61 of the SNPs are common among ND0501C, ND05-03B, NE06-02D, and NE06-02F (Supplementary Table S5). Hence, across the first strains, there are no identifiable polymorphisms within the thaxtomin PAIs, at the single nucleotide or gene level, that are candidates for explaining variations in virulence phenotypes.

\section{The genomes of the five strains are highly conserved in composition.}

Genes outside of the thaxtomin PAI and implicated in virulence have been previously identified and may potentially explain the phenotypic variation observed (Bignell et al. 2010b; Joshi et al. 2007a; Seipke and Loria 2008). Homologs of the $c e b R$ gene, which encodes a cellobiose sensor (Francis et al.
2015), are present in all five strains and are identical in sequence. Moreover, among the five strains, there are no nucleotide differences in predicted CebR boxes located within the thaxtomin PAIs. The presence or absence of other genes implicated in virulence does not correlate with a difference in virulence on radish or potato, as none of the five strains have identifiable homologs of NEC1, TOMA, or a coronafacic biosynthetic gene cluster. Therefore, we compared overall genetic distance in the context of a phylogenetic tree constructed on the basis of SNP differences (Fig. 5). ND05-03B is closely related, with no more than 1,465 SNP differences, to aggressive pathogenic strains (Fig. 5A; Supplementary Table S6). Notably, ND05-03B has only 795 SNP differences in comparison with ND05-1C. In contrast, ND05-13A is the most diverged of the five strains, with between 4,463 and 4,709 SNP differences relative to the other strains (Supplementary Table S6).

Regarding genome composition, the strains show few differences within the clade. Approximately $90 \%$ of the genes are core to all five strains. In fact, in all possible pairwise comparisons, strains exceed $95 \%$ conserved proteins. Strain ND0503B lacks homologs of only 65 genes, of which homologs are present in the other four thaxtomin-producing strains (Fig. 5B; Supplementary Table S8). In addition, the genome of ND0503B has the highest number of small INDELS (deletions relative to reference NE06-02D), ranging in size from 1 to $12,521 \mathrm{bp}$, in its genome (Supplementary Table S7). However, $72 \%$ of these INDELs are also detected in the most closely
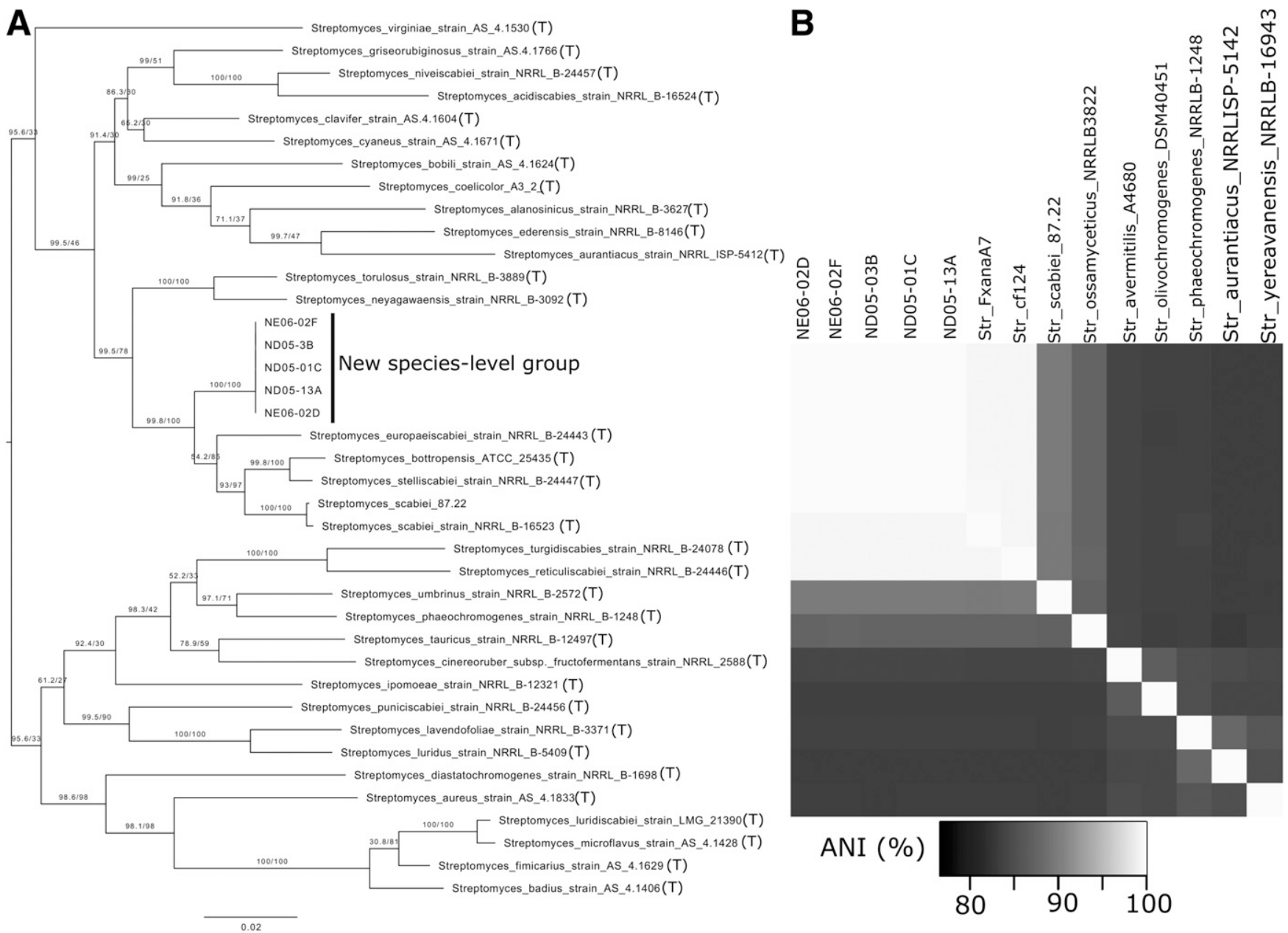

Fig. 3. Ribotype 3,6,7 strains form a novel species-level group. A, Multilocus sequence analysis-based maximum likelihood phylogeny of five strains studied here and select Streptomyces type strains that represent 11 known phytopathogenic species. (T) indicates type strains. B, A subset of a heatmap of all pairwise comparisons of average nucleotide identity, showing only the five newly sequenced strains and their most closely related strains. 
related and thaxtomin-producing strain, ND05-01C. In fact, $98.5 \%$ of the genomes of ND05-03B and ND05-01C are homologous, with only 269 INDELs. Strain ND05-13A, which is more distantly related to other strains, lacks homologs of 252 genes conserved in the three strains pathogenic on potato. Except for those annotated as phage or transposase related, most of these polymorphic genes are annotated as hypothetical proteins and do not provide insights into possible functions.

The genotypes and phenotypes of the strains are summarized in Table 2.

\section{DISCUSSION}

In this work, we i) employed genomics to circumscribe strains into a new species-level group of phytopathogenic Streptomyces strains, ii) characterized radish and potato plant

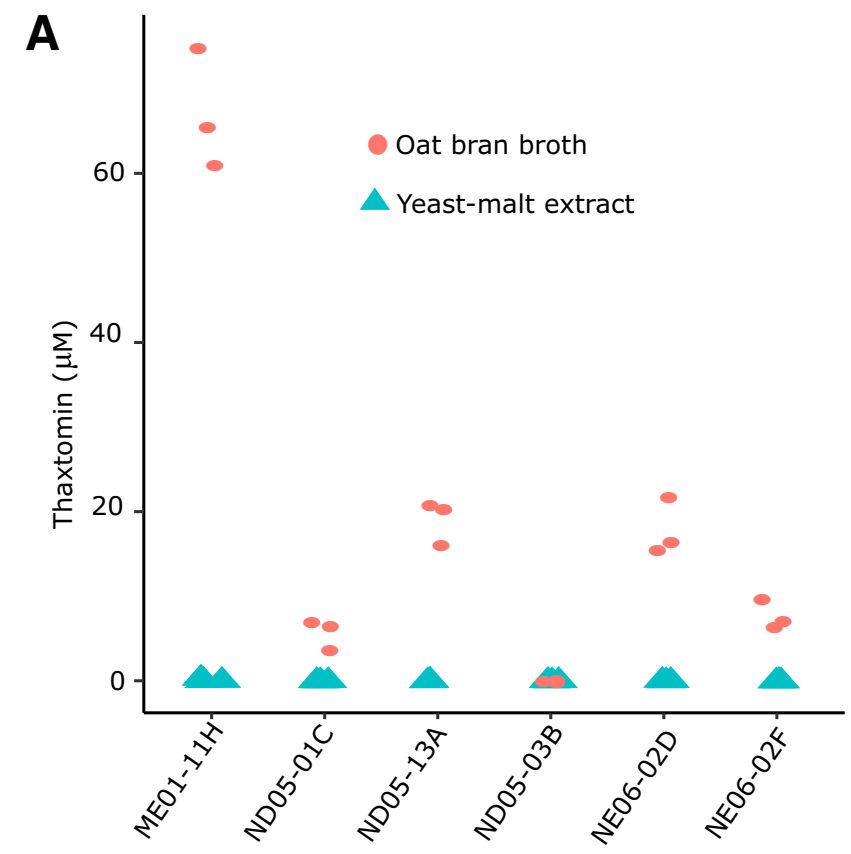

B

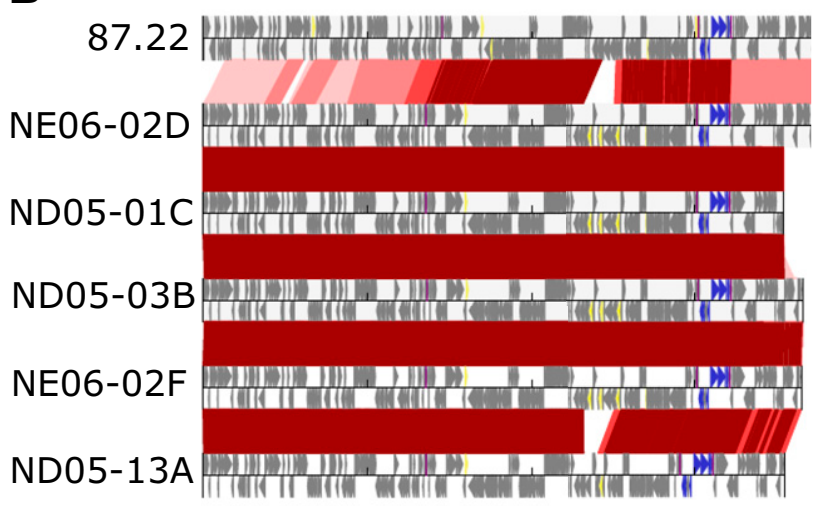

Fig. 4. The five strains have a $t x t$ pathogenicity island (PAI) but produce different levels of thaxtomin in culture. A, Thaxtomin amounts from Streptomyces strains grown for 7 days in either thaxtomin-inducing oat bran broth media (circles) or non thaxtomin-inducing yeast-malt extract (triangles). Data points are the average of two technical replicates for each of three flasks for each strain-media combination. B, Alignment of the thaxtomin biosynthetic cluster of the new species strains and that of S. scabiei 87.22. Blue arrows, $t x t$ genes; yellow arrows, insertion sequence elements; gray arrows, other genes; purple lines, att (flanking attachment) sites that are the predicted sites of integration of subregions of the thaxtomin PAI. Color shading indicates nucleotide identity with dark burgundy being $100 \%$ identity. pathogenicity and thaxtomin production by several strains in this novel species-level group, iii) identified a strain that is not pathogenic and does not produce detectable amounts of thaxtomin, despite encoding a complete thaxtomin biosynthetic gene cluster, and iv) identified a strain that is unable to cause significant common scab symptoms on potato, despite being able to produce thaxtomin and cause disease in radish.

Findings demonstrate that, even among closely related strains of the same species, there is substantial undescribed diversity that impacts the ability of a Streptomyces strain to become phytopathogenic. Additionally, the results demonstrate that the presence of the thaxtomin biosynthetic locus is not sufficient for pathogenicity and that there are undiscovered potato-specific virulence factors of phytopathogenic Streptomyces strains. These conclusions challenge the current paradigm of Streptomyces-plant interactions and are foundational for a mechanistic understanding of common scab and applying findings to better manage disease.

Also foundational is to develop a robust evolutionary framework for informing on and distinguishing between vertical and horizontal patterns in inheritance in plant pathogenic Streptomyces strains to better estimate the risk of disease emergence. Constructing phylogenies reflective of the evolutionary history of Streptomyces spp. is a critical first step. The combined use of multiple gene loci and analysis of wholegenome sequences yields a more accurate definition of Streptomyces species groups (Fig. 3; Supplementary Fig. S5) than analysis from 16S rDNA sequences alone (Supplementary Fig. S4), similar to previous studies of Streptomyces (Labeda 2011; Labeda et al. 2012). The use of lower resolution methods to classify Streptomyces species can introduce confusion, as exemplified by several Streptomyces strains named as S. scabiei not clustering with the S. scabiei type strain (Supplementary Fig. S3). These results further support previous suggestions that the taxonomy of plant-associated Streptomyces spp. should be revisited (Labeda et al. 2012; Thapa et al. 2019; Zhang et al. 2016).

By genotyping and phenotyping each Streptomyces strain individually, we revealed remarkable diversity in plant pathogenicity within this newly defined species-level group. There is little diversity among the five strains, which are closely related at a level of a species, and their genomes encode complete thaxtomin biosynthetic gene clusters (Fig. 4B). But, surprisingly, strain ND05-03B was unable to produce thaxtomin (Fig. $4 \mathrm{~A}$ ) and was unable to cause disease on radish or potatoes (Figs. 1 and 2). The inability of ND05-03B to produce thaxtomin and cause common scab in the tested conditions could not be attributed to the genetic sequence of the thaxtomin PAI. The thaxtomin biosynthetic PAI is identical between strains ND0503B and ND05-01C and only distinguished by two SNPs between ND05-03B and NE06-02F or NE06-02D (Supplementary Table S5). Therefore, we conclude that genes outside of the thaxtomin PAI are responsible for the observed differences in thaxtomin production among the tested strains. Artificial introduction of the thaxtomin PAI was previously shown to be insufficient to lead to thaxtomin production in multiple nonpathogenic Streptomyces species (Zhang et al. 2018); therefore, genes outside of the PAI affecting thaxtomin production is not particular to the novel species group described here. Identification of these critical genes outside of the thaxtomin PAI is potentially a more tractable problem using the strains tested here due to their high genetic similarly. These results also demonstrate that detection of the thaxtomin biosynthetic gene cluster is insufficient for accurate identification of common scab pathogens for all Streptomyces spp.

There were no obvious polymorphisms in the thaxtomin PAI that explain the inability of strain ND05-13A to cause 
significant disease on potato. While strain ND05-13A encodes a divergent PAI compared with the other characterized strains in the new species group, it is only divergent from the PAI of strain 87.22 by a single SNP. But strain 87.22 is a successful pathogen of potato while ND05-13A is not. Moreover, strain ND05-13A is able to produce thaxtomin in liquid media. We, therefore, conclude that the inability of ND05-13A to cause disease in potato is due to undiscovered potato-specific virulence factors or divergent regulation of thaxtomin production in strain ND05-13A on potato. At present, the genes important to the phenotypic variation discovered in this work are unknown. While there are only 95 predicted genes found in the genomes of the three analyzed pathogenic strains that are lacking in ND05-13A (Fig. 4), predicting the genes involved in the phenotypic variation is challenging due to the poor annotation of Streptomyces genomes. No genes known to be involved in the regulation of thaxtomin were identified as polymorphic among the strains.

One of the characterized strains (ND05-13A) was highly aggressive on radish but only weakly virulent on potato. From this observation, we suggest that the radish seedling assay, which is commonly used to assess Streptomyces plant pathogenicity, may not be a sufficient proxy for predicting potato virulence for all strains. Additionally, we found more significant stunting of radish seedlings when the Streptomyces strains were grown in thaxtomin-inducing $\mathrm{OB}$ medium than nonthaxtomin inducing YME medium (Fig. 1; Supplementary Fig. S1). This difference may be partially due to residual thaxtomin in the mycelial inoculums following growth in OB medium.

This work highlights the importance of understanding the diversity of plant-associated Streptomyces spp. The number of species able to cause common scab of potato likely remains underestimated. Additional genomic and phenotypic characterizations of Streptomyces isolates will likely reveal even more novel phenotypes and phytopathogenic species. Additional genomic-scale data for potato-associated Streptomyces strains will also further clarify the diversity of the thaxtomin PAI. The unknown virulence-associated genes that reside outside the thaxtomin PAI may also be identified through further genome sequencing and phenotypic characterization. A more complete

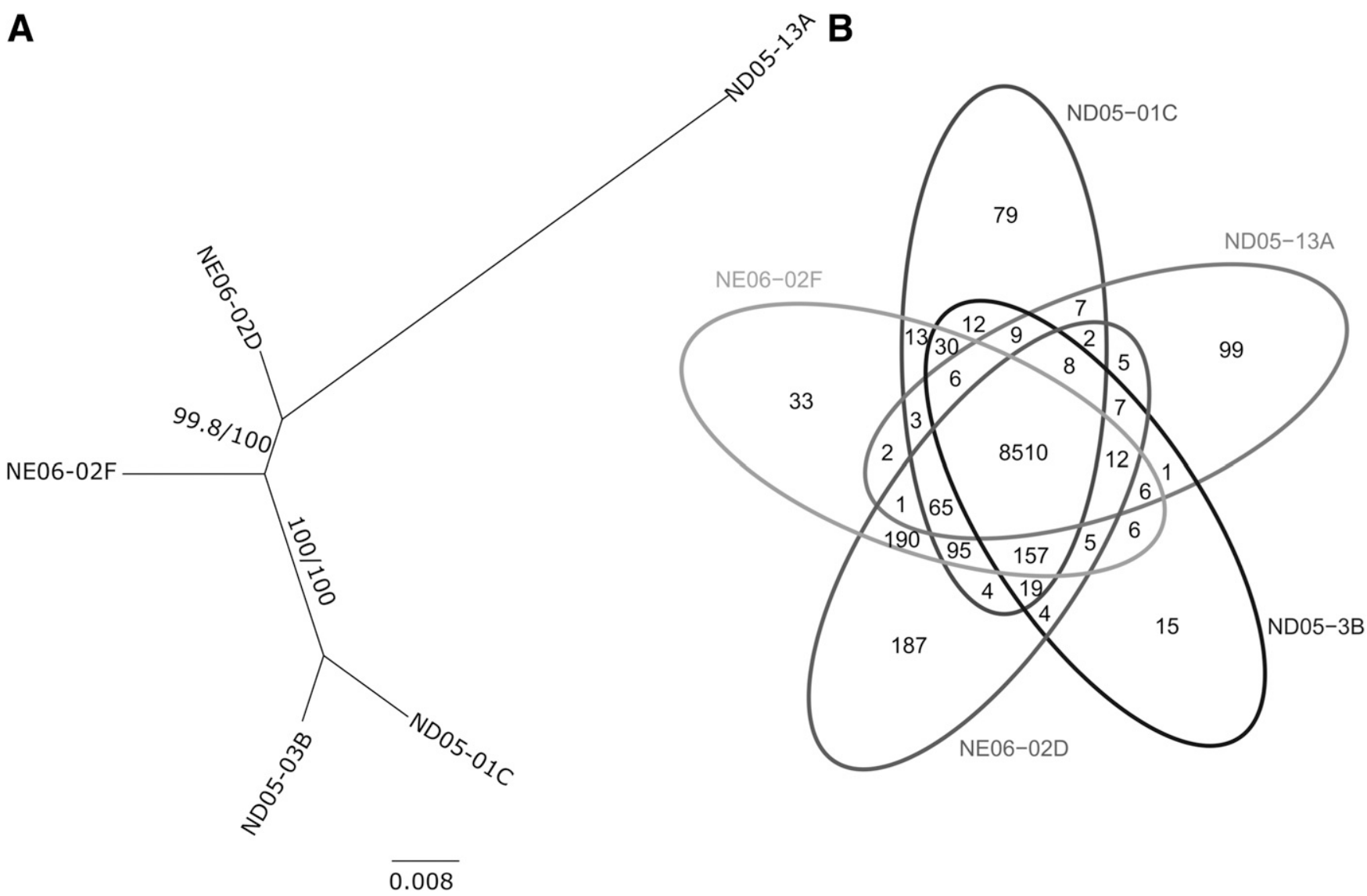

Fig. 5. The five analyzed strains are highly similar in genome composition. A, Maximum likelihood single nucleotide polymorphism-based phylogeny of ribotype 3,6,7 strains. The genome sequence of strain NE06-02D was used as a reference. The tree is midpoint rooted, branch labels indicate ultrafast bootstrap (UFBoot) and SH-aLRT test support as a percentage of 1,000 replicates each. B, Venn diagram of homologous and unique genes among the five strains. Annotated gene sequences were extracted, translated, and clustered on the basis of sequence homology.

Table 2. Summary of phenotypes and genotypes for tested strains of the new species group

\begin{tabular}{lccccccc}
\hline Strain name & Radish virulence & Potato virulence & Thaxtomin production & TXT locus & NECI & TOMA & Coronafacic acid \\
\hline ND05-01C & Aggressive & Aggressive & Yes & Yes & No & No & No \\
ND05-03B & None & None & No & Yes & No & No & No \\
ND05-13A & Aggressive & Very weak & Yes & No & No & No \\
NE06-02D & Aggressive & Aggressive & Yes & Yes & No & No \\
NE06-02F & Aggressive & Aggressive & Yes & Yes & No & No \\
\hline
\end{tabular}


understanding of the diversity of phytopathogenic Streptomyces spp. will improve the power of diagnostic assays for predicting common scab pressure in potato fields. Additionally, further characterization of the virulence mechanisms that underpin symptom development will enable identification of the potato genes responsible for cultivar-specific common scab resistance.

\section{MATERIALS AND METHODS}

\section{Radish necrosis assay.}

Radish necrosis assays were carried out using a protocol modified from one previously described (Bignell et al. 2010b). Radish cv. White Icicle (Ferry Morse) seeds were sterilized with $6 \%$ hydrogen peroxide for $10 \mathrm{~min}$ before draining and rinsing with $4 \%$ sodium hypochlorite. Seeds were immediately triple rinsed in double-distilled (dd) $\mathrm{H}_{2} \mathrm{O}$, were resuspended in $5 \mathrm{ml}$ of $\mathrm{ddH}_{2} \mathrm{O}$, were sowed onto water agar plates, and were placed in the dark at $28^{\circ} \mathrm{C}$ for 1 day. Twelve-well plates $(\mathrm{Co}-$ star) were filled ( $5 \mathrm{ml}$ per well) with water agar $(0.4 \%)$. Germinated radish seedlings were transferred into the wells containing the water agar. In parallel, Streptomyces strains were cultured on OB (Beauséjour et al. 1999) agar plates for 5 to 10 days until sporulation. Spores were sterilely harvested in $\mathrm{ddH}_{2} \mathrm{O}$, were quantified using a Leitz Dialux 22 microscope and hemocytometer, and were then diluted to a final concentration of 2,000 spores per microliter in $30 \mathrm{ml}$ of either OB media or YME media in a 250-ml Erlenmeyer flask. Cultures were grown in the flasks for 3 days at $28^{\circ} \mathrm{C}$, shaking at $200 \mathrm{rpm}$. The media containing the mycelial growth of the Streptomyces strains were transferred to pre-weighed centrifuge tubes, and mycelia were pelleted by centrifugation, using an Eppendorf $5810 \mathrm{R}$, at $4,000 \mathrm{rpm}$ at $4^{\circ} \mathrm{C}$ for $15 \mathrm{~min}$. The mycelial mass was weighed and diluted $1: 1$ with $\mathrm{dd}_{2} \mathrm{O}$. A total of $30 \mu \mathrm{l}$ of mycelial suspensions were then applied to germinated radish seedings in the 12-well plates. Plates containing the radish seedlings were covered and were transferred to a plant growth chamber (Percival LED41L2) at $29^{\circ} \mathrm{C}$ and a 12 -h light cycle. Radish seedlings were grown for 9 days and the number of true leaves, the hypocotyl length, and the root length of each radish seedling were measured. ANOVA and Tukey's honest significant difference (HSD) tests were performed in $\mathrm{R}$ version 3.6.0.

\section{Greenhouse potato pathogenicity assay.}

Potato pathogenicity assays were performed as previously described (Clarke et al. 2019), with slight modifications. Briefly, Streptomyces strains were grown on OB plates until sporulation. Spores were sterilely harvested and were used to inoculate $30 \mathrm{ml}$ of YME media in a 250-ml Erlenmeyer flask, and were grown for 3 days at $28^{\circ} \mathrm{C}$ and $230 \mathrm{rpm}$ shaking. Mycelial biomass was then harvested by centrifugation, was weighed, and was diluted to $0.3 \mathrm{~g} / \mathrm{ml}$. The mycelial suspension $(1 \mathrm{ml})$ was used to inoculate approximately $30 \mathrm{~g}$ of sterilized vermiculite in $8-\times 12$-in autoclave bags (VWR) along with $50 \mathrm{ml}$ of $2 \times$ Say's solution ( $40 \mathrm{~g}$ of sucrose, $2.4 \mathrm{~g}$ of asparagine, $1.2 \mathrm{~g}$ of $\mathrm{K}_{2} \mathrm{HPO}_{4}, 20 \mathrm{~g}$ of yeast extract per liter of $\mathrm{ddH}_{2} \mathrm{O}$ ). Streptomyces strains were grown in the vermiculite for 14 days, with manual kneading every 2 to 3 days. The concentration of Streptomyces strains in each bag was determined by sampling vermiculite, vortexing in sterile water, and dilution-plating on YME plates. To inoculate greenhouse-grown potato plants, vermiculite was weighed out to obtain $2 \times 10^{7}$ CFUs for each pot and was mixed using a Hobart commercial mixer, with 1:1 ratio of sterilized sand to sterilized potting mix (MetroMix Sungro 830). Seed tuber pieces were cut 2 days before planting, to induce suberization, and were then planted approximately $2 \mathrm{~cm}$ below the surface of the soil. Daughter tubers were harvested 12 weeks after planting and each tuber was individually scored for predominant lesion type (superficial, raised, or pitted) and percentage of total coverage of lesion. The final disease score is calculated by multiplying the percent total coverage by a lesion severity score, where the lesion severity score is 1 for superficial lesions, 3 for raised lesions, and 6 for pitted lesions. Clarke et al. (2020) discuss advantages of this scoring system to the classic scoring system based on the Horsfall Barett system (Haynes et al. 2010). For analysis, the eight replicate pots for each plant-pathogen combination (across multiple experimental blocks, due to greenhouse space limitations) were combined. ANOVA and Tukey's HSD tests were performed in $\mathrm{R}$ version 3.6.0.

\section{Extraction of Streptomyces DNA and whole- genome sequencing.}

Streptomyces strains ND05-01C, ND05-13A, NE06-02D, NE06-02F, and ND05-03B were cultured on YME (4 g of yeast extract, $10 \mathrm{~g}$ of malt extract, and $4 \mathrm{~g}$ of dextrose per liter of $\mathrm{dd}_{2} \mathrm{O}$ ) agar (1.7\%) plates for 8 to 12 days until sporulation. Spores were sterilely harvested in $3 \mathrm{ml}$ of $\mathrm{ddH}_{2} \mathrm{O}$ and were added to $30 \mathrm{ml}$ of liquid YME in a 250-ml Erlenmeyer flask. Cultures were then grown for 2 days at $28^{\circ} \mathrm{C}$ and $200 \mathrm{rpm}$ shaking. Mycelia were harvested by centrifugation and were resuspended 1:5 in sterile $50 \mathrm{mM}$ EDTA, $\mathrm{pH}$ 8.0. Total genomic DNA extraction was performed by following the gram-positive protocol of the Wizard Genomic DNA extraction kit (Promega) with lysozyme pretreatment $(10 \mathrm{mg} / \mathrm{ml}, 25 \mathrm{mM}$ sodium acetate, $\mathrm{pH} 4.5,50 \%$ glycerol).

Seqwell libraries were prepared from DNA samples and were sequenced (150 bp paired end) in multiplex with other libraries, on one lane of an Illumina HiSeq 3000 (Center for Genome Research and Biocomputing [CGRB], Corvallis, OR, U.S.A.). Previously reported methods were used to process and assemble sequencing reads and annotate assemblies (Savory et al. 2020; Weisberg et al. 2020). Briefly, reads were processed for quality and to remove adapter sequences and were de novo assembled, using SPAdes, into contigs (Bankevich et al. 2012). Prokka was used to annotate the assembled genome sequences (Seemann 2014).

FastANI v. 1.1 was used to calculate pairwise ANI between genome sequences (Jain et al. 2018). The R packages gplots 3.0.1 and viridis v. 0.5.1 were used to plot a heatmap of ANI values (Garnier et al. 2018). Streptomyces genome sequences were downloaded from the National Center for Biotechnology Information (NCBI) on April 20, 2019. Accession numbers are listed in Supplementary Table S3.

Get_homologs v. 3.2.2 with MCL clustering and the options "-n 1 -M -t 0 -m cluster -s -P" was used to cluster genes from the five sequenced strains into orthologous groups (ContrerasMoreira and Vinuesa 2013). The Get_homologs script parse_ pangenome_matrix.pl was used to identify genes that are present in a group of strains and absent from another. The $\mathrm{R}$ script overLapper was used to generate a Venn diagram of shared gene content among strains based on the ortholog clustering analysis (Backman and Girke 2016).

MLSA was performed using autoMLSA with the options “-entrez_query 'Streptomyces[ORGANISM]' -remote refseq genomic -nocomplete -prog tblastn" and the protein sequences of AtpD, GyrB, RecA, RpoB, and TrpB (Davis et al. 2016; Labeda 2011). IQTREE v. 1.6.12 with the options "-bb 1000 -alrt 1000 " was used to generate a phylogenetic tree (Nguyen et al. 2015). Phylogenies were plotted using FigTree v. 1.4.4 or the R package ggtree (available on figtree) (Yu et al. 2017). Protein sequences were downloaded from NCBI on November 18, 2019.

Whole genome SNP analysis was performed using a modified version of the GATK best practices. BWA v. 0.7.17-r1188 with the options "mem -M" was used to map paired reads to the 
genome sequence of NE06-02D (Li and Durbin 2009). Sam alignments were sorted and indexed and duplicates were marked, using Broad Institute's Picard tools v. 2.0.1 (2019). SNPs were called individually for each strain, using GATK Haplotype caller, with the options "-ERC GVCF -ploidy 1", and were combined using GenotypeGVCFs with the default options (McKenna et al. 2010). SNPs were filtered using the GATK tool VariantFiltration with filters QD $<18$, SOR $>3$, QUAL <30, FS >60, MQ <50, MQRankSum $<-12.5$, and ReadPosRankSum $<-8$, followed by the SelectVariants tool. $\mathrm{Vcf}$ files were converted to fasta format using the vcftools $\mathrm{v}$. 0.1 .16 program vcf-to-tab followed by the vcftab_to_fasta_ alignment.pl script (J. Chen personal communication; Danecek et al. 2011). IQTREE v. 1.6.12 with the options "-m GTR+ASC -bb 1000 -alrt 1000" was used to generate a phylogenetic tree. SnpEff v. 4.3t with the options "-no-downstream -no-upstream -no-intron" was used to predict effects of SNPs on gene functions (Cingolani et al. 2012).

The program lastz v. 1.04.01 was used to compare contigs containing the PAI region (Harris 2007). Three runs for each pairwise comparison were performed, with similarity searches in both directions and the option "-format=BLASTN", as well as a more stringent search with the options "-format=BLASTN -exact=12 -nogapped -filter=identity:95", to identify highly similar regions. Blastn and settings "-word_size 7 -evalue 1000 " was used to search for the sequence "TTGAAGCGGAAC" to identify the location of PAI borders. Only full identity matches were considered. Gene maps showing homologous regions larger than $1 \mathrm{~kb}$ were generated using the BioPython tool GenomeDiagram (Pritchard et al. 2006).

\section{High-performance liquid chromatography (HPLC) quantification of thaxtomin.}

Streptomyces strains were cultured on $\mathrm{OB}$ agar plates until sporulation. Spores were sterilely harvested in $\mathrm{ddH}_{2} \mathrm{O}$, were quantified using a hemocytometer, and were then added to $30 \mathrm{ml}$ of either OBB or YME media in 250-ml flasks, to a final concentration of 2,000 spores/ $\mu$. Three replicate cultures were grown for each strain-media combination. Cultures were then grown for 7 days at $28^{\circ} \mathrm{C}$ and $200 \mathrm{rpm}$ shaking. Cultures were pelleted for $1 \mathrm{~h}$ at $4^{\circ} \mathrm{C}$ and $4,000 \mathrm{rpm}$ and supernatants were transferred and stored at $-20^{\circ} \mathrm{C}$, until use. Total thaxtomin present in each sample was quantified using an Agilent 1200 HPLC system coupled with an Agilent 1200 system coupled with diode array (Agilent) and single quad mass spectrometer detector (Thermo Fisher Scientific MSQ Plus) instruments operated using Chromeleon software (Thermo Fisher Scientific). Liquid chromatography-mass spectrometry (MS) analysis was carried out with a slightly modified method as described previously (Clarke et al. 2019). In brief, an Agilent Eclipse Plus C18 column $(1.8 \mathrm{um}, 4.6 \times 50 \mathrm{~mm})$ equipped with a Phenomenex guard column (Security Guard) with C18 cartridge was used for chromatographic separation. Flow gradient was as follows: 0 to $4 \mathrm{~min}, 30$ to $95 \% \mathrm{~B} ; 4$ to $5 \mathrm{~min}, 95 \% \mathrm{~B} ; 5$ to $5.1 \mathrm{~min}, 95$ to $30 \% \mathrm{~B} ; 5.1$ to $6 \mathrm{~min}, 30 \% \mathrm{~B}$. The run time was $6 \mathrm{~min}$, and the ultraviolet lamp was set at $\lambda \max 240 \mathrm{~nm}$ and $380 \mathrm{~nm}$. Only wavelength $380 \mathrm{~nm}$ was used to quantify thaxtomin. MS was used for the molecular weight confirmation only. MSQ conditions were: ESI +ve mode, Mass range $\mathrm{m} / z, 100$ to 1,000 , scan time $1 \mathrm{~s}$, cone voltage $70 \mathrm{~V}$, probe temperature $300^{\circ} \mathrm{C}$. Full scan $(\mathrm{m} / \mathrm{z}, 100$ to 1,000$)$ and SIM for $\mathrm{m} / \mathrm{z}$ 439. The developed method was validated for linearity range, precision, the limit of detection, the limit of quantification, and accuracy, for thaxtomin as per International Council for Harmonization of Technical Requirements for Pharmaceuticals for Human Use (2005) guidelines.

\section{ACKNOWLEDGMENTS}

We thank K. Haynes (Agricultural Research Service [ARS]) for providing Chippewa, Green Mountain, and Elkton seed tubers and thank R. Novy (ARS) for providing the Payette Russet seed tubers. We thank the CGRB for their services and the Department of Botany and Plant Pathology for supporting the computing infrastructure.

\section{AUTHOR-RECOMMENDED INTERNET RESOURCES}

Broad Institute's Picard toolkit: http://broadinstitute.github.io/picard FigTree: http://tree.bio.ed.ac.uk/software/figtree

CRAN-R gplot tools:

https://cran.r-project.org/web/packages/gplots/index.html

LPSN: www.bacterio.net

vcftab_to_fasta_alignment.pl script:

https:/github.com/JinfengChen/vcf-tab-to-fasta

\section{LITERATURE CITED}

Backman, T. W. H., and Girke, T. 2016. systemPipeR: NGS workflow and report generation environment. BMC Bioinformatics 17:388.

Bankevich, A., Nurk, S., Antipov, D., Gurevich, A. A., Dvorkin, M., Kulikov, A. S., Lesin, V. M., Nikolenko, S. I., Pham, S., Prjibelski, A. D., Pyshkin, A. V., Sirotkin, A. V., Vyahhi, N., Tesler, G., Alekseyev, M. A., and Pevzner, P. A. 2012. SPAdes: A new genome assembly algorithm and its applications to single-cell sequencing. J. Comput. Biol. 19: 455-477.

Beauséjour, J., Goyer, C., Vachon, J., and Beaulieu, C. 1999. Production of thaxtomin A by Streptomyces scabies strains in plant extract containing media. Can. J. Microbiol. 45:764-768.

Bignell, D. R. D., Francis, I. M., Fyans, J. K., and Loria, R. 2014 Thaxtomin A production and virulence are controlled by several bld gene global regulators in Streptomyces scabies. Mol. Plant-Microbe Interact. 27:875-885.

Bignell, D. R. D., Huguet-Tapia, J. C., Joshi, M. V., Pettis, G. S., and Loria, R. 2010a. What does it take to be a plant pathogen: Genomic insights from Streptomyces species. Antonie van Leeuwenhoek 98: 179-194.

Bignell, D. R. D., Seipke, R. F., Huguet-Tapia, J. C., Chambers, A. H., Parry, R. J., and Loria, R. 2010b. Streptomyces scabies 87-22 contains a coronafacic acid-like biosynthetic cluster that contributes to plantmicrobe interactions. Mol. Plant-Microbe Interact. 23:161-175.

Bouchek-Mechiche, K., Gardan, L., Andrivon, D., and Normand, P. 2006. Streptomyces turgidiscabies and Streptomyces reticuliscabiei: One genomic species, two pathogenic groups. Int. J. Syst. Evol. Microbiol. 56:2771-2776

Braun, S., Gevens, A., Charkowski, A., Allen, C., and Jansky, S. 2017. Potato common scab: A review of the causal pathogens, management practices, varietal resistance screening methods, and host resistance. Am. J. Potato Res. 94:283-296.

Bukhalid, R. A., and Loria, R. 1997. Cloning and expression of a gene from Streptomyces scabies encoding a putative pathogenicity factor. J. Bacteriol. 179:7776-7783.

Bukhalid, R. A., Takeuchi, T., Labeda, D., and Loria, R. 2002. Horizontal transfer of the plant virulence gene, nec1, and flanking sequences among genetically distinct Streptomyces strains in the Diastatochromogenes cluster. Appl. Environ. Microbiol. 68:738-744.

Cingolani, P., Platts, A., Wang, L., Coon, M., Nguyen, T., Wang, L., Land, S. J., Lu, X., and Ruden, D. M. 2012. A program for annotating and predicting the effects of single nucleotide polymorphisms, SnpEff: SNPs in the genome of Drosophila melanogaster strain w1118; iso-2; iso-3. Fly (Austin) 6:80-92.

Clarke, C. R., Kramer, C. G., Kotha, R. R., Wanner, L. A., Luthria, D. L., and Kramer, M. 2019. Cultivar resistance to common scab disease of potato is dependent on the pathogen species. Phytopathology 109:1544-1554.

Clarke, C. R., Tegg, R. S., Thompson, H. K., Frederick, C., Haynes, K. G., Kramer, M., and Wilson, C. R. 2020. Low-dose foliar treatments of the auxin analog 2,4-D reduce potato common scab and powdery scab for multiple potato cultivars and enhance root development. Crop Prot. 136: 105208.

Contreras-Moreira, B., and Vinuesa, P. 2013. GET_HOMOLOGUES, a versatile software package for scalable and robust microbial pangenome analysis. Appl. Environ. Microbiol. 79:7696-7701.

Danecek, P., Auton, A., Abecasis, G., Albers, C. A., Banks, E., DePristo, M. A., Handsaker, R. E., Lunter, G., Marth, G. T., Sherry, S. T., McVean, 
G., and Durbin, R. 2011. The variant call format and VCFtools. Bioinformatics 27:2156-2158.

Davis, E. W., II Weisberg, A. J., Tabima, J. F., Grunwald, N. J., and Chang, J. H. 2016. Gall-ID: Tools for genotyping gall-causing phytopathogenic bacteria. PeerJ 4:e2222.

Francis, I., Holsters, M., and Vereecke, D. 2010. The gram-positive side of plant-microbe interactions. Environ. Microbiol. 12:1-12.

Francis, I. M., Jourdan, S., Fanara, S., Loria, R., and Rigali, S. 2015. The cellobiose sensor CebR is the gatekeeper of Streptomyces scabies pathogenicity. MBio 6:e02018-14.

Fry, B. A., and Loria, R. 2002. Thaxtomin A: Evidence for a plant cell wall target. Physiol. Mol. Plant Pathol. 60:1-8.

Garnier, S., Ross, N., Rudis, B., Sciaini, M., and Scherer, C. (2018). viridis: Default Color Maps from 'matplotlib'. CRAN-R. https://cran.rproject.org/web/packages/viridis/index.html.

Harris, R. S. 2007. Improved pairwise alignment of genomic DNA. The Pennsylvania State University, State College, PA, U.S.A.

Haynes, K. G., Wanner, L. A., Thill, C. A., Bradeen, J. M., Miller, J., Novy, R. G., Whitworth, J. L., Corsini, D. L., and Vinyard, B. T. 2010. Common scab trials of potato varieties and advanced selections at three U.S. locations. Am. J. Potato Res. 87:261-276.

Healy, F. G., Wach, M., Krasnoff, S. B., Gibson, D. M., and Loria, R. 2000. The txtAB genes of the plant pathogen Streptomyces acidiscabies encode a peptide synthetase required for phytotoxin thaxtomin A production and pathogenicity. Mol. Microbiol. 38:794-804.

Jain, C., Rodriguez-R, L. M., Phillippy, A. M., Konstantinidis, K. T., and Aluru, S. 2018. High throughput ANI analysis of $90 \mathrm{~K}$ prokaryotic genomes reveals clear species boundaries. Nat. Commun. 9:5114.

Johnson, E. G., Joshi, M. V., Gibson, D. M., and Loria, R. 2007. Cellooligosaccharides released from host plants induce pathogenicity in scabcausing Streptomyces species. Physiol. Mol. Plant Pathol. 71:18-25.

Joshi, M., Rong, X., Moll, S., Kers, J., Franco, C., and Loria, R. 2007a. Streptomyces turgidiscabies secretes a novel virulence protein, Nec1, which facilitates infection. Mol. Plant-Microbe Interact. 20:599-608.

Joshi, M. V., Bignell, D. R. D., Johnson, E. G., Sparks, J. P., Gibson, D. M., and Loria, R. 2007b. The AraC/XylS regulator TxtR modulates thaxtomin biosynthesis and virulence in Streptomyces scabies. Mol. Microbiol. 66:633-642.

Jourdan, S., Francis, I. M., Deflandre, B., Loria, R., and Rigali, S. 2017. Tracking the subtle mutations driving host sensing by the plant pathogen Streptomyces scabies. MSphere 2:e0367-16.

Kers, J. A., Cameron, K. D., Joshi, M. V., Bukhalid, R. A., Morello, J. E., Wach, M. J., Gibson, D. M., and Loria, R. 2005. A large, mobile pathogenicity island confers plant pathogenicity on Streptomyces species. Mol. Microbiol. 55:1025-1033.

Labeda, D. P. 2011. Multilocus sequence analysis of phytopathogenic species of the genus Streptomyces. Int. J. Syst. Evol. Microbiol. 61: 2525-2531.

Labeda, D. P., Dunlap, C. A., Rong, X., Huang, Y., Doroghazi, J. R., Ju, K.-S., and Metcalf, W. W. 2017. Phylogenetic relationships in the family Streptomycetaceae using multi-locus sequence analysis. Antonie van Leeuwenhoek 110:563-583.

Labeda, D. P., Goodfellow, M., Brown, R., Ward, A. C., Lanoot, B., Vanncanneyt, M., Swings, J., Kim, S. B., Liu, Z., Chun, J., Tamura, T., Oguchi, A., Kikuchi, T., Kikuchi, H., Nishii, T., Tsuji, K., Yamaguchi, Y., Tase, A., Takahashi, M., Sakane, T., Suzuki, K. I., and Hatano, K. 2012. Phylogenetic study of the species within the family Streptomycetaceae. Antonie van Leeuwenhoek 101:73-104.

Lerat, S., Simao-Beaunoir, A.-M., Wu, R., Beaudoin, N., and Beaulieu, C. 2010. Involvement of the plant polymer suberin and the disaccharide cellobiose in triggering thaxtomin A biosynthesis, a phytotoxin produced by the pathogenic agent streptomyces scabies. Phytopathology 100:91-96.

Li, H., and Durbin, R. 2009. Fast and accurate short read alignment with Burrows-Wheeler transform. Bioinformatics 25:1754-1760.
Loria, R., Bignell, D. R. D., Moll, S., Huguet-Tapia, J. C., Joshi, M. V., Johnson, E. G., Seipke, R. F., and Gibson, D. M. 2008. Thaxtomin biosynthesis: The path to plant pathogenicity in the genus Streptomyces. Antonie van Leeuwenhoek 94:3-10.

McKenna, A., Hanna, M., Banks, E., Sivachenko, A., Cibulskis, K., Kernytsky, A., Garimella, K., Altshuler, D., Gabriel, S., Daly, M., and DePristo, M. A. 2010. The genome analysis toolkit: A MapReduce framework for analyzing next-generation DNA sequencing data. Genome Res. 20:1297-1303.

Nguyen, L.-T., Schmidt, H. A., von Haeseler, A., and Minh, B. Q. 2015. IQTREE: A fast and effective stochastic algorithm for estimating maximum-likelihood phylogenies. Mol. Biol. Evol. 32:268-274.

Pritchard, L., White, J. A., Birch, P. R. J., and Toth, I. K. 2006. GenomeDiagram: A python package for the visualization of large-scale genomic data. Bioinformatics 22:616-617.

Rückert, C., Albersmeier, A., Busche, T., Jaenicke, S., Winkler, A., Friðjónsson, Ó. H., Hreggviðsson, G. Ó., Lambert, C., Badcock, D., Bernaerts, K., Anne, J., Economou, A., and Kalinowski, J. 2015. Complete genome sequence of Streptomyces lividans TK24. J. Biotechnol. 199:21-22.

Sarwar, A., Latif, Z., and Cabaleiro, C. 2018. First report of Streptomyces bottropensis causing potato common scab in Galicia, Spain. Plant Dis. 102:1445.

Savory, E. A., Weisberg, A. J., Stevens, D. M., Creason, A. L., Fuller, S. L., Pearce, E. M., and Chang, J. H. 2020. Phytopathogenic Rhodococcus have diverse plasmids with few conserved virulence functions. Front. Microbiol. 11:1022.

Seemann, T. 2014. Prokka: Rapid prokaryotic genome annotation. Bioinformatics 30:2068-2069.

Seipke, R. F., and Loria, R. 2008. Streptomyces scabies 87-22 possesses a functional tomatinase. J. Bacteriol. 190:7684-7692.

Studholme, D. J. 2016. Genome update. Let the consumer beware: Streptomyces genome sequence quality. Microb. Biotechnol. 9:3-7.

Thapa, S. P., Davis, E. W., 2nd, Lyu, Q., Weisberg, A. J., Stevens, D. M., Clarke, C. R., Coaker, G., and Chang, J. H. 2019. The evolution, ecology, and mechanisms of infection by gram-positive, plant-associated bacteria. Annu. Rev. Phytopathol. 57:341-365.

Wach, M. J., Krasnoff, S. B., Loria, R., and Gibson, D. M. 2007. Effect of carbohydrates on the production of thaxtomin A by Streptomyces acidiscabies. Arch. Microbiol. 188:81-88.

Wanner, L. A. 2004. Field isolates of streptomyces differ in pathogenicity and virulence on radish. Plant Dis. 88:785-796.

Wanner, L. A. 2006. A survey of genetic variation in streptomyces isolates causing potato common scab in the United States. Phytopathology 96: 1363-1371.

Wanner, L. A. 2009. A patchwork of streptomyces species isolated from potato common scab lesions in North America. Am. J. Potato Res. 86: 247-264.

Weisberg, A.J., Davis, E.W., Tabima, J., Belcher, M.S., Miller, M., Kuo, C.-H., Loper, J.E., Grünwald, N.J., Putnam, M.L., and Chang, J.H. 2020. Unexpected conservation and global transmission of agrobacterial virulence plasmids. Science 368:eaba5256.

Yu, G., Smith, D. K., Zhu, H., Guan, Y., and Lam, T. T.-Y. 2017. ggtree: An $\mathrm{R}$ package for visualization and annotation of phylogenetic trees with their covariates and other associated data. Methods Ecol. Evol. 8:28-36.

Zhang, Y., Bignell, D. R. D., Zuo, R., Fan, Q., Huguet-Tapia, J. C., Ding, Y., and Loria, R. 2016. Promiscuous pathogenicity islands and phylogeny of pathogenic Streptomyces spp. Mol. Plant-Microbe Interact. 29: 640-650.

Zhang, Y., Jiang, G., Ding, Y., and Loria, R. 2018. Genetic background affects pathogenicity island function and pathogen emergence in Streptomyces. Mol. Plant Pathol. 19:1733-1741.

Zhou, B., Zhang, M. S., and Ma, X. K. 2017. First report of Streptomyces bottropensis causing potato common scab in Hebei Province, China. Plant Dis. 101:502. 